\title{
Optimization of a frame structure subjected to a plastic deformation
}

\author{
K. Yuge \\ Faculty of Engineering, Seikei University, Kichi-joji Kitamachi 3, Musashino-shi, Tokyo, Japan
}

\section{N. Kikuchi}

Department of Mechanical Engineering and Applied Mechanics, The University of Michigan, Ann Arbor, MI 48109, USA

\begin{abstract}
An optimization method for a frame structure subjected to a plastic deformation is proposed in this paper. The method is based on the generalized layout optimization method proposed by Bendsøe and Kikuchi in 1988, where the solid-cavity composite material is distributed in the admissible domain and the cavity size is determined so that it becomes large in the area where the strain energy is small. Elasto-plastic analysis based on the homogenization method is carried out to obtain the nonlinear average stress-strain relations of a porous material first. Then the optimization algorithm of a frame structure is derived by taking plastification into account. Finally in order to demonstrate the effectiveness of the present algorithm, several numerical examples are illustrated.
\end{abstract}

\section{Introduction}

The purpose of structural analysis is mainly to reconfirm the safety of a structure planned by well-experienced designers. It can predict where the damage will take place by the applied load but does not give a better design itself. The development of structural optimization analysis, however, has changed this role of structural analysis to a very active one in design. Among optimization methods, sizing optimization is the simplest method where sizes of structural members are determined by sensitivity analysis. Another method is shape optimization in which outline curves of specified structural members are determined. Haftka and Grandhi (1986) made a detailed survey of this method. The design obtained by sizing or shape optimization analysis, however, may not optimal since they do not change the topology of the initial design and it is generally very difficult to know the optimal topology prior to sizing or shape optimization analysis.

Bendsøe and Kikuchi (1988) proposed a new approach to the topological and shape optimization of linear elastic structures, which they named the generalized layout optimization method. In this theory, the admissible domain is assumed to be filled with microstructures with cavities. The sizes of cavities and the rotational angle of microstructures are determined with a constraint of a given amount of material so that the cavities may become larger as the strain energy is relatively smaller. One of the main features is that the homogenization method is employed to determine the effective average elastic constants of the microstructures. The finite element implementation of the homogenization method for the linear elastic problem was discussed by Guedes and Kikuchi (1990). The generalized layout optimization method given by Bendsøe and Kikuchi (1988) was justified through many numerical examples by Suzuki and Kikuchi (1991) with studies of the effect of support and loading conditions on the solution. Diaz and Kikuchi (1992) extended the algorithm for the dynamic problem in which the fundamental eigenvalue of a structure is maximized by reinforcement using a prescribed amount of material. Ma et al. (1993) modified the iterative method based on the conventional optimality criteria for more general dynamic problems.

Due to the research mentioned above, the generalized layout optimization method has developed rapidly and is advancing in linear elastic static or dynamic structural problems. In this paper, the algorithm is applied to the optimal design of frame structures subjected to plastic deformations. Although there has been extensive research on the optimal design of frame structures, not much work related to plastic deformations has been carried out. It is, however, important in designing a frame structure to maximize the energy absorption capacity by a plastic deformation to avoid its fatal collapse by an earthquake. Here, we treat such problems in that we shall distribute a prescribed amount of the material optimally inside frame members so that the external work done by the prescribed load may become minimum. As a result, some domains in structural members may be hollow. To discretize a frame structure, the linear Timoshenko beam element proposed by Hughes (1977) is employed with the socalled layered approach, where multilayers are assumed in the transverse direction. Each layer is assumed to be composed of a periodic microstructure with a square cavity whose size we shall define by sensitivity analysis. The average uniaxial stress-stain relations of the present material should be known as a function of the cavity size in sensitivity analysis. We calculate these relations by the elasto-plastic finite element analysis based on the homogenization method.

In the following sections the algorithm of the elasto-plastic finite element analysis based on the homogenization method is briefly discussed and numerical examples are shown initially. Secondly, the average stress-strain relations of the present material are calculated for various cavity sizes and the relations obtained are approximated as a simple function of the cavity size for sensitivity analysis. Then the algorithm 
of optimal analysis for a frame structure subjected to a plastic deformation is described, and finally several numerical examples of optimization analysis are shown to demonstrate the effectiveness of the present algorithm.

2 Elasto-plastic finite element analysis based on the homogenization method

As previously mentioned, elasto-plastic finite element analysis based on homogenization is conducted in this paper to obtain the nonlinear stress strain relations of the solid-cavity material. The algorithm of the finite element analysis for linear elastic materials based on the homogenization method was discussed precisely by Guedes and Kikuchi (1990). Here the algorithm is extended to elasto-plastic analysis. In the present formulation, the following assumptions are employed.

- The stress field is the plane stress.

- The bilinear isoparametric element is employed to discretize both the global domain and a microstructure

- Plastic deformations of each constitutive material inside a microstructure follow the plastic flow theory

- Finite deformations in the global domain are considered with the updated Lagrangian formulation while it is neglected in a microstructure

In the last assumption, we limit our objective problems to those in which deformations in a microstructure are small. This is mainly because we are not interested in the problem of local instability in a microstructure. Local instability may take place especially in those microstructurs that have a fairly large void, which remains unsolved even in the elastic problems.

Assume a two-dimensional problem in which a structure is composed of a periodical microstructure as shown in Fig. 1.

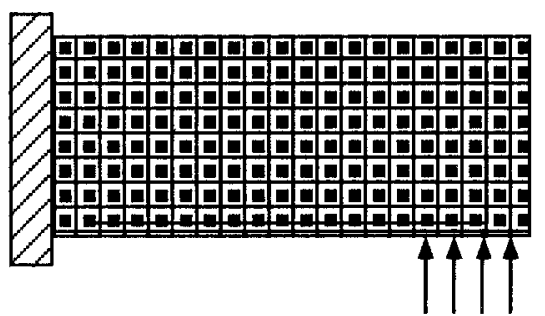

Fig. 1. Two-dimensional structure composed of a periodical microstructure

If the microstructure can be regarded as infinitely small, we can calculate effective material constants by the homogenization method. The material constants may be different throughout the whole structure due to plastification in the elasto-plastic analysis, while they are independent of the global position and loading history in the elastic analysis. It is not a practical idea, however, to calculate these constants in the whole domain. Here the average material constants are evaluated at every Gaussian quadrature point in a "global" element and stresses in a microstructure are also evaluated at the quadrature points. Figure 2 shows the discretized global domain and a discretized microstructure at a quadrature point. The dots in Fig. 2 shows the Gaussian quadrature points of a global element.

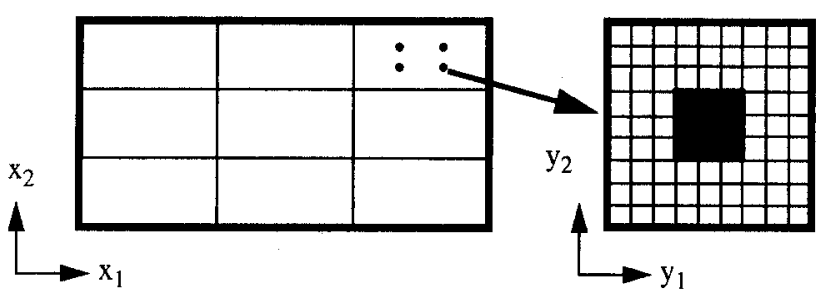

Fig. 2. Discretization of the global domain and a microstructure

\subsection{Stiffness equations of the global domain}

In the elasto-plastic analysis, the incremental method is generally employed. The principle of the virtual work between the $n$-th and the $(n+1)$-th incremental steps for the global domain $\Omega$ is give in an incremental form as

$\int_{\Omega}\left[\delta \Delta \varepsilon_{g} \Delta \sigma_{g}+\delta \Delta \varepsilon_{g}^{*} \sigma_{g}^{(n)}\right] \mathrm{d} \Omega=\left(\delta \Delta \mathbf{u}_{g}\right) \Delta \mathbf{f}_{g}$

where $\delta$ expresses variation, $\Delta \varepsilon_{g}, \Delta \varepsilon_{g}^{*}$ are the linear and quadratic strain increments, $\sigma_{g}^{(n)}$ represents the average stresses within the microstructure at the $n$-th step, respectively, and $\Delta \mathbf{f}_{g}$ and $\Delta \mathbf{u}_{g}$ are external load increments and corresponding displacement increments. We define the following strain-displacement matrix $\mathbf{B}$ and the stress-strain matrix $\mathbf{D}^{H}$ as:

$\Delta \varepsilon_{g}=\mathbf{B} \Delta \mathbf{u}_{g}^{e}, \quad \Delta \sigma_{g}=\mathbf{D}^{H} \Delta \varepsilon_{g}$,

where $\mathbf{u}_{g}^{e}$ are the displacements of a global element, and $\mathbf{D}^{H}$ is composed of the average or homogenized material constants, the evaluation of which is shown in the next section. Substituting (2) into (1), the stiffness equations of the global domain are derived as follows:

$\sum_{i=1}^{N_{e g}} \mathbf{K}_{g} \Delta \mathbf{u}_{g}^{e}=\sum_{i=1}^{N_{e g}} \Delta \mathbf{f}_{g}^{e}, \quad \mathbf{K}_{g}=\int_{\Omega_{e}}\left(\mathbf{B}^{t} \mathbf{D}^{H} \mathbf{B}+\mathbf{K}_{0}\right) \mathrm{d} \Omega$,

where $N_{e g}$ is the number of the global elements, $\Omega_{e}$ is the element domain, $\mathbf{f}_{g}^{e}$ expresses the element forces, and $\boldsymbol{K}_{0}$ is the initial stress matrix derived from the second term on the left-hand side of (1). Once the element displacement increments $\Delta \mathbf{u}_{g}^{e}$ are obtained by solving (3), the average stress increments at Gaussian quadrature points should be calculated by (2) and added to the stresses at $n$-th step as

$\sigma_{g}^{(n+1)}=\sigma_{g}^{(n)}+\Delta \sigma_{g}$,

since these are necessary to calculate the initial stress matrix $\mathbf{K}_{0}$ in (3) at the next incremental step. Finally the nodal coordinates should be updated.

\subsection{Evaluation of the average material constants}

It is necessary to evaluate $\mathbf{D}^{H}$ to compose the stiffness (3). The algorithm is briefly described here based on the algorithm given by Guedes and Kikuchi (1990).

Consider that a porous material formed by a periodic microstructure whose representative length is very small, of order $\varepsilon$ is subjected to external force. Entreating the higher order terms, the displacement at $(\mathbf{x}, \mathbf{y})$ can be expressed as the sum of the global and the microscopic displacement as $\mathbf{u}^{\varepsilon}=\mathbf{u}^{0}(\mathbf{x})+\varepsilon \mathbf{u}^{1}(\mathbf{x}, \mathbf{y})$,

where 
$u_{i}^{1}(\mathbf{x}, \mathbf{y})=-\chi_{i}^{k \ell}(\mathbf{x}, \mathbf{y}) \frac{\partial u_{k}^{0}(\mathbf{x})}{\partial x_{\ell}}+u_{i}(\mathbf{x}) \quad(i, k, \ell=1,2)$.

Here $\mathbf{x}$ are the global coordinates, $\mathbf{y}$ are the local coordinates of the microstructure and $\mathbf{y}=\mathbf{x} / \varepsilon \cdot \mathbf{u}(\mathbf{x})$ are arbitrary additive constants in $\mathbf{y} ; \chi^{k \ell}$ are the solutions

$\int_{Y} \mathbf{D}_{i j p m} \frac{\partial \chi_{p}^{k \ell}}{\partial y_{m}} \frac{\partial \nu_{i}(\mathbf{y})}{\partial y_{j}} \mathrm{~d} Y=\int_{Y} \mathbf{D}_{i j k \ell} \frac{\partial \nu_{i}(\mathbf{y})}{\partial y_{j}} \mathrm{~d} Y$,

$\forall \nu \in V_{Y} \quad(i, j, p, m=1,2)$,

$V_{Y}=\left\{\nu \in \mathbf{H}^{1} \mid \quad \nu\right.$ is $Y$ periodic in the unit cell $\}$,

respectively. Here $\mathbf{H}^{\mathbf{1}}$ is the Sovolev space, and $\mathbf{Y}=(-1 / 2$, $-1 / 2) \times(1 / 2,1 / 2)$ in the $\mathbf{y}=\{y 1, y 2\}$ coordinate system, and $\mathbf{D}_{i j k \ell}$ is the material tensor. The homogenized material tensor is given as

$\mathbf{D}_{i j k \ell}^{H}=\frac{1}{|Y|} \int_{Y}\left(\mathbf{D}_{i j k \ell}-\mathbf{D}_{i j p m} \frac{\partial \chi_{p}^{k \ell}}{\partial y_{m}}\right) \mathrm{d} Y$.

Since $\mathbf{D}_{i j k \ell}$ is a function of stress history in the elasto-plastic analysis, the stresses inside a microstructure should be calculated. From (5) and (6), the displacement increments can be expressed as

$\Delta u_{i}^{1}=-\chi_{i}^{k \ell}(x, y) \frac{\partial \Delta u_{k}^{0}}{\partial x_{\ell}} \quad(i, k, \ell=1,2)$.

The stress increments in a local element are evaluated as $\Delta \sigma_{i j(\ell)}=\mathbf{D}_{i j k m}\left(\frac{\partial \Delta u_{k}^{0}}{\partial x_{m}}+\frac{\partial \Delta u_{k}^{1}}{\partial y_{m}}\right) \quad(i, j, k, m=1,2)$.

If the material is elastic, $\mathbf{D}_{i j k \ell}$ is

$\mathbf{D}_{i j k \ell}=\mathbf{D}_{i j k \ell}^{e}$,

where $\mathbf{D}_{i j k \ell}^{e}$ is the linear elastic constant for the plane stress field. If the material is plastic, it can be expressed as

$\mathbf{D}_{i j k \ell}=\mathbf{D}_{i j k \ell}^{e}-\frac{\left(\mathbf{D}_{i j p q}^{e} a_{p q}\right)\left(a_{r s} \mathbf{D}_{r s k \ell}^{e}\right)}{H^{\prime}+a_{p q} \mathbf{D}_{p q r s}^{e} a_{r s}}$

where $H^{\prime}$ is the strain hardening modulus and is expressed with the elastic modulus $E$ and the tangential modulus $E_{t}$ as

$H^{\prime}=\frac{E E_{t}}{E-E_{t}}$,

and $a_{r s}$ is defined as

$a_{r s}=\frac{\partial f}{\partial \sigma_{r s}}$,

where $f$ is the flow potential and is chosen as the von Mises yield criterion. The calculated stress increments $\Delta \sigma_{\ell}$ given in (10) are added to the stresses at the present incremental stresses as

$\sigma_{i j(\ell)}^{(n+1)}=\sigma_{i j(\ell)}^{(n)}+\Delta \sigma_{i j(\ell)}$.

The number of sampling points to evaluate stresses in an element may depend on the problem. Here we employ four sampling points identical to the Gaussian quadrature points.

Since a microstructure is very small compared with the global domain, the stress increments $\Delta \sigma_{\ell}$ may be fairly large even if the global displacement increments are not so large. As a result, the equivalent stress may be much larger than the yield stress and cause a significant error. Therefore it is preferable to employ the techniques proposed by Owen and Hinton (1980) to avoid stresses drifting away from the yield surface.

Figure 3 shows the flow chart of the present algorithm.

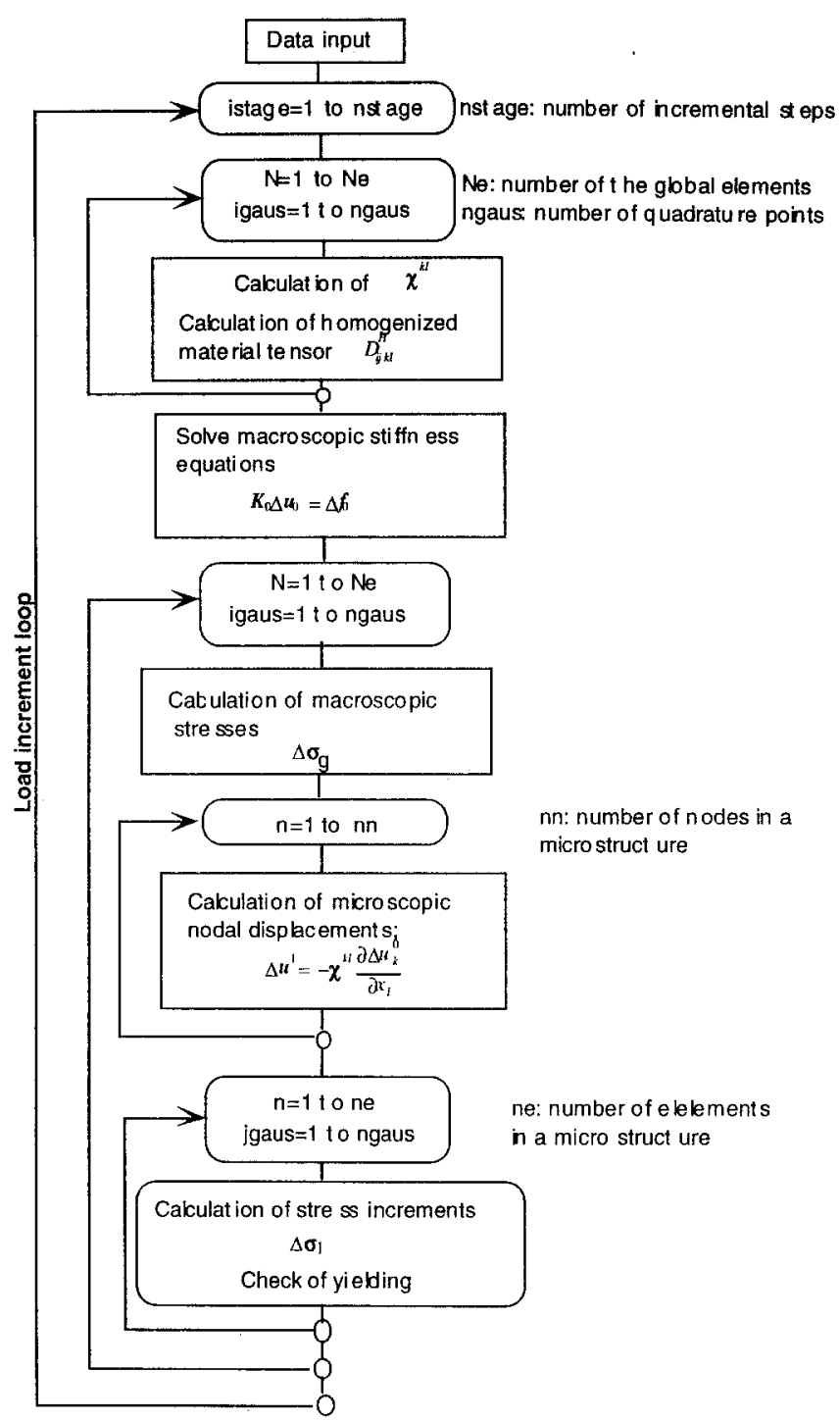

Fig. 3. Elasto-plastic FEA of the homogenization method

2.3 Numerical example of the elasto-plastic analysis of the homogenization method

To examine the present algorithm, the plastic buckling analysis of a simply supported beam as shown in Fig. 4a was calculated. The beam was assumed to be composed of an infinitely small periodic square microstructure which has a square cavity in it as shown in Fig. 4b. The direction of the $y$-coordinate system is identical to the $x$-coordinate system initially. The material properties are the elastic modulus $E$ $=206 \mathrm{GPa}$, Poisson's ratio $\nu=0.3$, the yield stress $\sigma_{y}=294$ $\mathrm{MPa}$ and the tangential modulus $E_{t}=10^{-4} E$ (constant). Due to symmetry, one half of the beam is divided to $20 \mathrm{el}-$ ements $(2 \times 10)$ and a prescribed displacement increment is given at one end. First it was confirmed that the results 
for $a=1$ (solid) agree well with those obtained by the conventional finite element analysis without the homogenization method. Then several cases for different values of $a$ were calculated. As the cavity size became smaller, i.e. $a$ went to 1 , the load-lateral displacement curve converged to that of the solid case. The load-lateral displacement curve for $a=$ 0.6 is shown compared with that for $a=1$ in Fig. 5, where $P$ is normalized by $P_{y}=\sigma_{y} t$. The deforming procedure is shown in Fig. 6. Although the displacement is fairly large, the calculated residual force is generally very small and the iteration was seldom required.

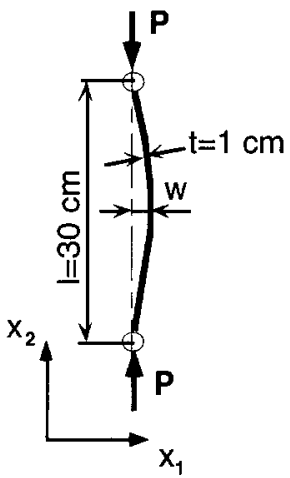

(a) Buckling of an simply supported beam

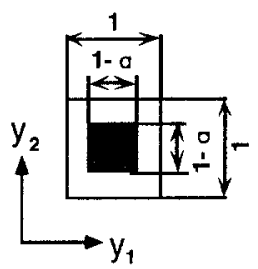

(b) Unit microstructure with a square cavity

Fig. 4. Elasto-plastic analysis based on the homogenization method

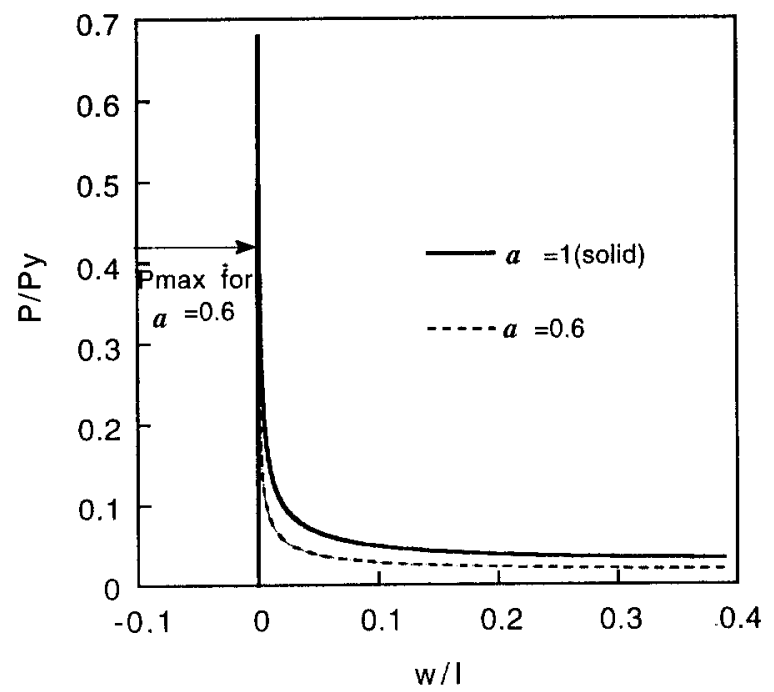

Fig. 5. Load-displacement relations of an axially compressed beam

\subsection{Stress-strain relation of the porous material}

As mentioned in the Introduction, we are interested in obtaining an optimal design of a frame structure subjected to a plastic deformation by use of the generalized layout optimization method proposed by Bendsøe and Kikuchi (1988), where the optimal topology and shape is expressed by varying the cavity size of the material. A detailed formulation of the present optimization problem will be given in the next section and here we will calculate the average stress-strain relations

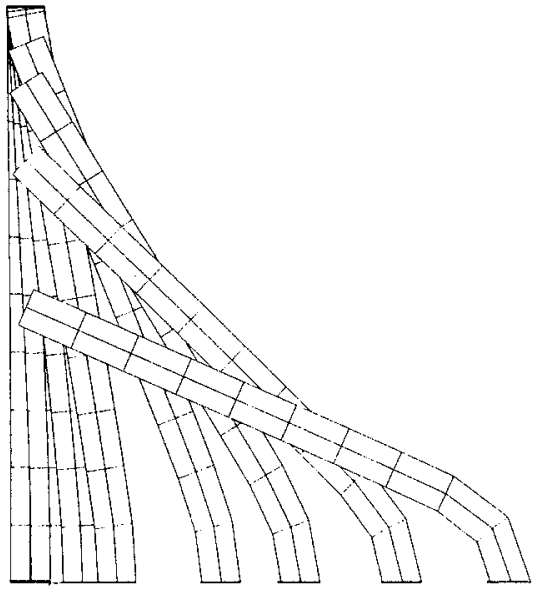

Fig. 6. Deforming process of an axially compressed beam

of the porous material composed of the uniform and periodic square microstructure shown in Fig. 7. These relations are necessary in the sensitivity analysis of a frame structure.

With the algorithm presented in the previous sections, the relations can be obtained by calculating the problem shown in Fig. 8 where the plate is assumed to be composed of the infinitely small periodic microstructure shown in Fig. 7. The material properties are the elastic modulus $E_{0}=206 \mathrm{GPa}$, Poisson's ratio $\nu=0.3$, the yield stress $\sigma_{y 0}=294 \mathrm{MPa}$ and the tangential modulus $E_{t}=0$.

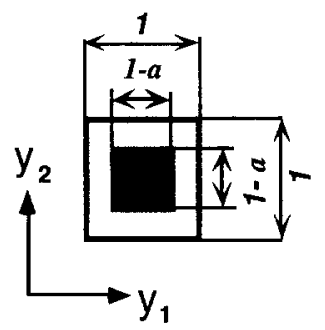

Fig. 7. Microstructure with a square cavity

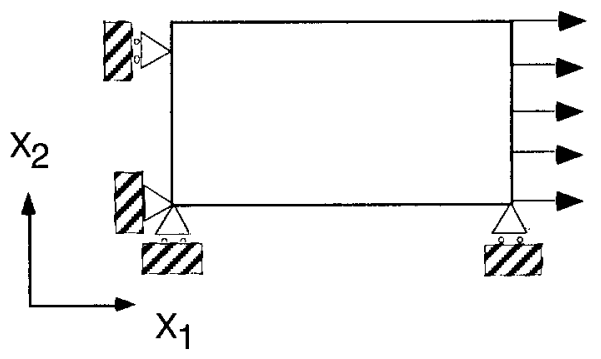

Fig. 8. A plate subjected to uniaxial tension

The average stress-strain relations obtained for various cavity sizes are depicted in Fig. 9 where the average stress is normalized by the yield stress $\sigma_{y 0}$, and the average strain is normalized by the yield strain $\varepsilon_{y 0}=\sigma_{y 0} / E_{0}$, respectively. The development of the area yielded within a microstructure for $a=0.2$ and $a=0.6$ is shown in Fig. 10. In the black elements plastic deformations took place at all the quadrature points and in the gray elements plastic deformations occurred at some of the quadratic points. It can be observed that the procedure of plastification is quite different in these two cases. 
From the average stress-strain curves obtained, the average elastic modulus $E_{0}^{H}$ is approximated as

$E_{0}^{H}(a)=\left(\frac{43}{40} a\right) E_{0} \quad(0 \leq a \leq 0.8)$,

$E_{0}^{H}(a)=\left(\frac{7}{10} a+\frac{3}{10}\right) E_{0} \quad(0.8<a \leq 1.0)$.

It would be better to set a very small value as the average tangential modulus $E_{t}^{H}(a)$ instead of zero for convenience. Here we employ the following value:

$E_{t}^{H}(a)=E_{0} / 1000$.

The relation between the calculated and the approximated elastic modulus is shown in Fig. 11. Observing the curves shown in Fig. 9, we can assume that the stress-strain curve is that of the elastic-perfectly plastic material and the initial yield strain is equal to $\varepsilon_{y 0}$ for every value of $a$, which results in the approximation of the average yield stress as

$\sigma_{y}^{H}(a)=\left(\frac{43}{40} a\right) \sigma_{y 0} \quad(0 \leq a \leq 0.8)$,

$\sigma_{y}^{H}(a)=\left(\frac{7}{10} a+\frac{3}{10}\right) \sigma_{y 0} \quad(0.8<a \leq 1.0)$.

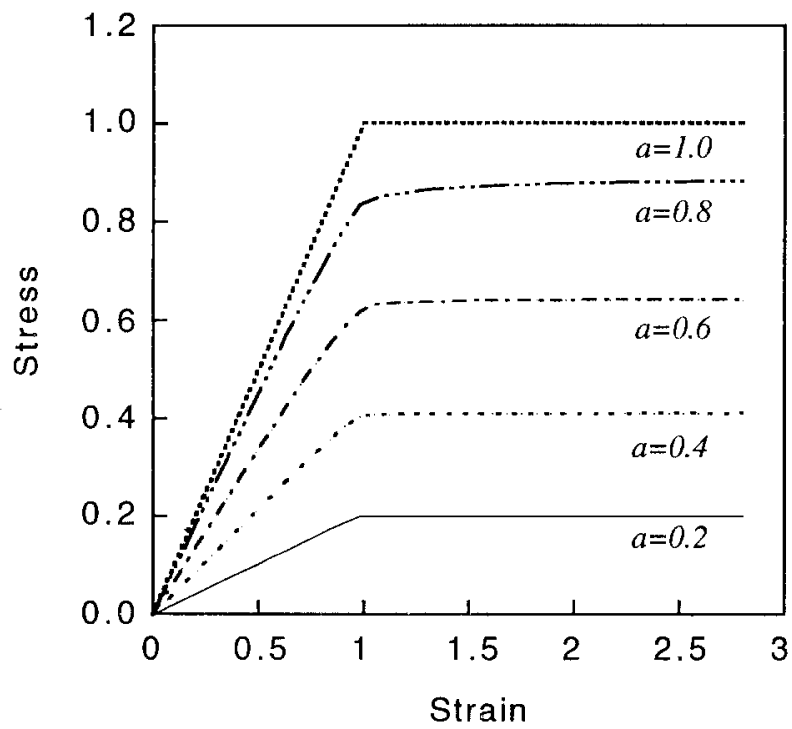

Fig. 9. Average stress-strain relations of the porous material subjected to uniform tension

\section{Optimization of a frame structure}

Here the algorithm of optimization analysis and numerical examples are presented. First the characteristics of a linear Timoshenko beam are briefly explained. Secondly, the objective function to be optimized and the sensitivity analysis are discussed. Finally numerical examples are illustrated.

\subsection{Linear Timoshenko beam element}

In the following formulation, the linear Timoshenko beam element shown in Fig. 12 with the layered approach is used to discretize a frame structure. The one point quadrature is employed to avoid locking. Each layer is assumed to be composed of a periodic square microstructure with a square hole

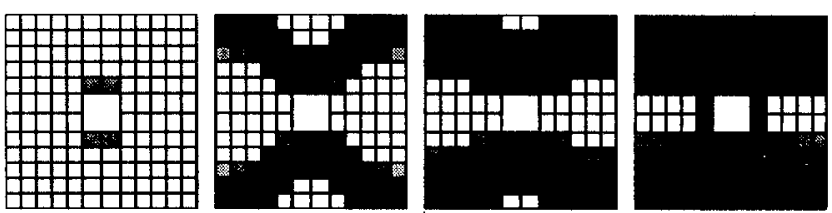

(a) $a=0.2$

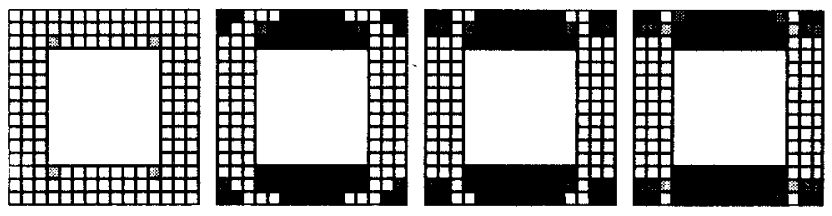

tension

(b) $a=0.6$

Fig. 10. Procedure of plastification in the microstructure

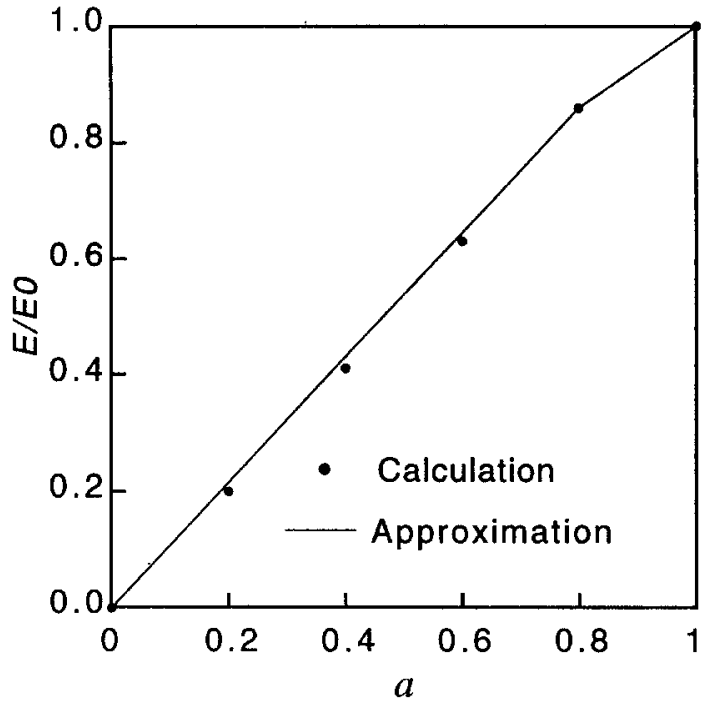

Fig. 11. Approximated homogenized Young's modulus

as shown in Fig. 13. We will optimize the material distribution in a frame structure by changing the hole size $a$ under a total volume constraint.

The normal and shear strains at $x=0$ and the distance of $z$ from the neutral axis are expressed with the nodal displacements and the element length $\ell$ as

$\varepsilon=\frac{u_{2}-u_{1}}{\ell}+z\left(\frac{\theta_{2}-\theta_{1}}{\ell}\right), \quad \gamma=\frac{\theta_{1}+\theta_{2}}{2}+\frac{w_{2}-w_{1}}{\ell}$

or in a matrix form

$\varepsilon=\mathbf{B}_{T} \mathbf{U}$,

where $\varepsilon=\lfloor\varepsilon, \gamma\rfloor$ and $U$ are the nodal displacements. We assume the stress-strain relations as

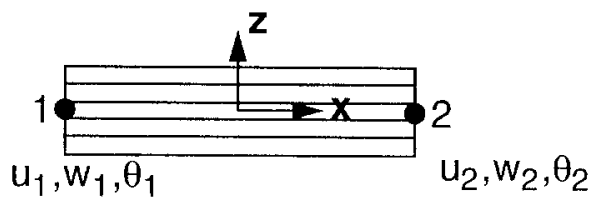

Fig. 12. Linear Timoshenko beam element with layers

$\Delta \sigma=E^{H}(a) \Delta \varepsilon, \quad \Delta \tau=G \Delta \gamma$,

and $E^{H}(a)$ is the average elasto-plastic modulus of the composite material while $G$ is the shear modulus and independent 


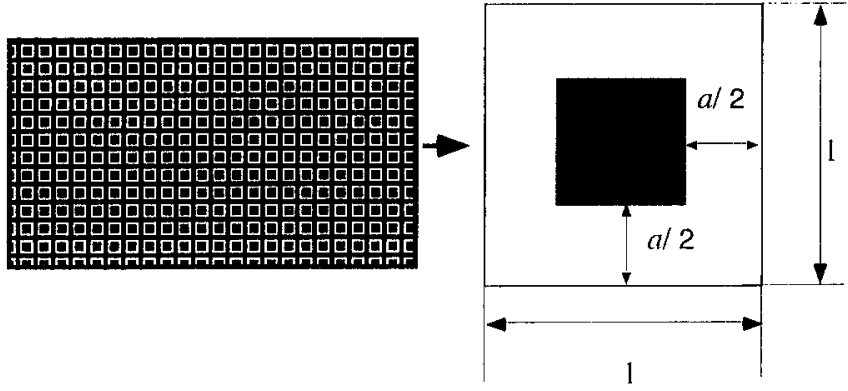

Fig. 13. Material with a periodic microstructure with a square hole

of the parameter $a$. We assume that there is the least material in frame members to prevent shear deformations and regard the design obtained as the optimal reinforcement. Although we may neglect the effect of the shear deformations for most of the frame structures, it would be better to consider the effect for those frame structures in which fairly short members are used. The formulation to consider the shear deformations will be discussed later

We can express $E^{H}(a)$ using the results obtained in 2.4 $E^{H}(a)=E_{0}^{H}(a) \quad$ (if the material is elastic), $E^{H}(a)=E_{t}^{H}(a) \quad$ (if the material is plastic),

(20) can be expressed in a matrix form

$\Delta \sigma=\mathbf{D} \Delta \varepsilon$,

where

$\Delta \sigma=\lfloor\Delta \sigma, \Delta \tau\rfloor$.

The incremental stiffness equations of the element are expressed as

$\mathbf{K} \boldsymbol{\Delta} \mathbf{u}=\mathbf{\Delta f}$,

where

$\mathbf{K}=\int_{\Omega} \mathbf{B}_{T}^{t} \mathbf{D B} \mathbf{B}_{T} \mathrm{~d} \Omega$,

and $\Delta \mathrm{u}$ and $\boldsymbol{\Delta} \mathbf{f}$ are the nodal displacement and the nodal force increments, respectively.

\subsection{Optimization algorithm of a frame structure}

Assume we optimize a frame structure so that the external work done by the applied force may be minimized. Our objective fuction would be

$F=\iint_{\Omega}^{u} \mathbf{f} \cdot \mathbf{d u d} \Omega$

where $\Omega$ is the structural domain, $\mathbf{u}$ is the nodal displacement vector and $\mathbf{f}$ is the external force vector. A volume constraint is

$h=\int_{\Omega}\left[1-(1-a)^{2}\right] \mathrm{d} \Omega-\Omega_{0} \leq 0$.

The optimization problem can be written minimize $F$ subject to $h(a) \leq 0, \underline{a}_{\ell}<a<\bar{a}_{\ell}$,

where $\underline{a}_{\ell}, \bar{a}_{\ell}$ stand for the permissible minimum and maximum values of the design variables $a$ and are identical to 0 and 1 , respectively. The Lagrangian function of the constrained optimization problem is defined as
$L=F+\lambda h+\sum_{\ell=1}^{N \ell}\left[\alpha_{\ell}\left(\underline{a}_{\ell}-a_{\ell}\right)+\beta_{\ell}\left(\bar{a}_{\ell}-a_{\ell}\right)\right]$,

where $\lambda$ and $\alpha_{\ell}, \beta_{\ell}$ are the Lagrange multipliers, and $N \ell$ is the number of layers. The Kuhn-Tucker conditions of the problem become

$\frac{\partial F}{\partial a_{\ell}}+\lambda \frac{\partial h}{\partial a_{\ell}}=\alpha_{\ell}-\beta_{\ell} \quad(\ell=1,2, \ldots, N \ell), \quad \lambda h=0$,

$\alpha_{\ell}\left(\underline{a}_{\ell}-a_{\ell}\right)=0 \quad(\ell=1,2, \ldots, N \ell)$,

$\beta_{\ell}\left(a_{\ell}-\bar{a} \ell\right)=0 \quad(\ell=1,2, \ldots, N \ell)$,

where

$\lambda \geq 0, \alpha_{\ell} \geq 0, \beta_{\ell} \geq 0 \quad(\ell=1,2, \ldots, N \ell)$.

The evaluation of the term $\partial F / \partial a_{\ell}$ is discussed later. According to the algorithm proposed by Bendsøe and Kikuchi (1988), $a_{\ell}(\ell=1,2, \ldots, N \ell)$ can be determined as follows.

(a) Give the initial value $a_{\ell}(\ell=1,2, \ldots, N \ell)$.

(b) Calculate $F$ by the elasto-plastic analysis.

(c) Calculate $\partial F / \partial a_{\ell}$ and $\partial h / \partial a_{\ell}$.

(d) Set the initial value of $\lambda^{k}$.

(e) Modify $a_{\ell}(\ell=1,2, \ldots, N \ell)$ as follows.

$a_{\ell}^{k+1}=$

$\begin{cases}\max \left[(1-\xi) a_{\ell}^{k}, \underline{a}_{\ell}\right] & \text { if } a_{\ell}^{k} D_{\ell}^{k} \leq \max \left[(1-\xi) a_{\ell}^{k}, \underline{a}_{\ell}\right] \\ a_{\ell}^{k} D_{\ell}^{k} & \text { if } \max \left[(1-\xi) a_{\ell}^{k}, \underline{a}_{\ell}\right]< \\ & a_{\ell}^{k} D_{\ell}^{k}<\min \left[(1+\xi) a_{\ell}^{k}, \bar{a}_{\ell}\right] \\ \min \left[(1+\xi) a_{\ell}^{k}, \bar{a}_{\ell}\right] & \text { if } a_{\ell}^{k} D_{\ell}^{k} \geq \min \left[(1+\xi) a_{\ell}^{k}, \bar{a}_{\ell}\right]\end{cases}$

where

$D_{\ell}^{k}=\left[-\left(\frac{\partial h}{\partial a_{\ell}} \lambda^{k}\right)^{-1} \frac{\partial F}{\partial a_{\ell}}\right]^{\eta}$,

and $\xi, \eta$ are a move limit and a weighing factor, respectively. (f) Modify $\lambda^{k}$ by the bisection method so that the following equation is satisfied:

$\int_{\Omega}\left\{1-\left[1-a^{k}\left(\lambda^{k}\right)\right]^{2}\right\} \mathrm{d} \Omega=\Omega_{0}$.

(g) Repeat (e)-(f) until $\lambda^{k}$ converges.

(h) Repeat (b)-(g) until a convergence criterion is satisfied

In the following we derive the term $\partial F / \partial a_{\ell}$ in the elastoplastic analysis.

In elasto-plastic analysis, the load-displacement relation is approximated to be piecewise linear and the load incremental method is applied. Using the incremental form, (8) is expressed as the summation of the external work increment done by the applied loads at each incremental step

$F=\sum_{i=1}^{N \text { stage }}\left(\mathbf{f}^{i} \Delta \mathbf{u}+\frac{1}{2} \Delta \mathbf{u} \Delta \mathbf{f}\right)$,

where $N$ stage and $i$ stand for the number of the incremental steps and the $i$-th incremental step, respectively. Taking differentiation with respect to the design variable $a_{\ell}$, we obtain the following equation:

$\frac{\partial F}{\partial a_{\ell}}=\sum_{i=1}^{N \text { stage }}\left(\mathrm{f}^{i} \frac{\partial \Delta \mathbf{u}}{\partial a_{\ell}}+\frac{1}{2} \Delta \mathbf{u} \frac{\partial \Delta \mathbf{f}}{\partial a_{\ell}}+\frac{1}{2} \Delta \mathbf{f} \frac{\partial \Delta \mathbf{u}}{\partial a_{\ell}}\right)$. 
Substituting the following relation obtained by differentiating the stiffness equations with respect to $a_{\ell}$ :

$\frac{\partial \mathbf{K}}{\partial a_{\ell}} \Delta \mathbf{u}+\mathbf{K} \frac{\partial \Delta \mathbf{u}}{\partial a_{\ell}}=0$,

we obtain

$\frac{\partial F}{\partial a_{\ell}}=\sum_{i=1}^{N \text { stage }}\left[\mathbf{f}^{i}\left(-\mathbf{K}^{-1} \frac{\partial \mathbf{K}}{\partial a_{\ell}} \Delta \mathbf{u}\right)+\right.$

$\left.\frac{1}{2} \Delta \mathbf{f}\left(-\mathbf{K}^{-1} \frac{\partial \mathbf{K}}{\partial a_{\ell}} \Delta \mathbf{u}\right)\right]=$

$-\sum_{i=1}^{N \text { stage }}\left[\mathbf{u}_{0}\left(\int_{\Omega} \mathbf{B}^{t} \frac{\partial \mathbf{D}}{\partial a_{\ell}} \mathbf{B} \mathrm{d} \Omega\right) \Delta \mathbf{u}+\right.$

$\left.\frac{1}{2} \Delta \mathbf{u}\left(\int_{\Omega} \mathbf{B}^{t} \frac{\partial \mathbf{D}}{\partial a_{\ell}} \mathbf{B} \mathrm{d} \Omega\right) \Delta \mathbf{u}\right]=$

$-\sum_{i=1}^{N \text { stage }} \int\left(\varepsilon_{0} \frac{\partial \mathbf{D}}{\partial a_{\ell}} \Delta \varepsilon+\frac{1}{2} \Delta \varepsilon \frac{\partial \mathbf{D}}{\partial a_{\ell}} \Delta \varepsilon\right) \mathrm{d} \Omega=$

$-\sum_{i=1}^{N \text { stage }} \int\left[\left(\frac{\partial \sigma^{i}}{\partial a_{\ell}}+\frac{1}{2} \frac{\partial \Delta \sigma}{\partial a_{\ell}}\right) \Delta \varepsilon\right] \mathrm{d} \Omega$.

Where the vectors $\mathbf{u}_{0}$ and $\varepsilon_{0}$ stand for

$\mathbf{u}_{0}=\mathbf{K}^{-1} \mathbf{f}^{i}, \quad \varepsilon_{0}=\mathbf{B} \mathbf{u}_{0}$,

respectively. Equation (37) can be rewritten as

$\frac{\partial F}{\partial a_{\ell}}=-\frac{\partial\left(\int_{\Omega} \int_{0}^{\varepsilon} \sigma \mathrm{d} \varepsilon \mathrm{d} \Omega\right)}{\partial a_{\ell}}=-\frac{\partial\left(\int_{\Omega} U_{\mathrm{int}} \mathrm{d} \Omega\right)}{\partial a_{\ell}}=$

$-t L_{e} \frac{\partial U_{\mathrm{int}}}{\partial a_{\ell}}$,

where $U_{\text {int }}, t, L_{e}$ are the internal work done throughout the whole loading procedure, and the thickness and length of the layer.

If we assume that deformations are small and strains increase monotonously in the present problems, we can derive $U_{\text {int }}$ at $\varepsilon=\left(\varepsilon_{1}, \gamma_{1}\right)$ by using of $(20)$ and (21) as

$U_{\text {int }}=\frac{1}{2} E_{0}^{H}\left(a_{\ell}\right) \varepsilon_{1}^{2}+\frac{1}{2} G \gamma_{1}^{2} \quad\left(\right.$ if $\left.\left|\varepsilon_{1}\right|<\varepsilon_{y 0}\right)$,

$U_{\text {int }}=\frac{1}{2} E_{0}^{H}\left(a_{\ell}\right) \varepsilon_{y 0}^{2}+E_{0}^{H}\left(a_{\ell}\right) \varepsilon_{y 0}\left(\left|\varepsilon_{1}\right|-\varepsilon_{y 0}\right)+$

$\frac{1}{2} E_{t}^{H}\left(\left|\varepsilon_{1}\right|-\varepsilon_{y 0}\right)^{2}+\frac{1}{2} G \gamma_{1}^{2} \quad$ (if $\left.\left|\varepsilon_{1}\right| \geq \varepsilon_{y 0}\right)$.

Thus, the differentiation of $U_{\text {int }}$ with respect to $a_{\ell}$, is obtained as

$\frac{\partial U_{\text {int }}}{\partial a_{\ell}}=\alpha E_{0} \frac{\varepsilon_{1}^{2}}{2} \quad\left(\right.$ if $\left.\left|\varepsilon_{1}\right|<\varepsilon_{y 0}\right)$,

$\frac{\partial U_{\text {int }}}{\partial a_{\ell}}=\alpha E_{0}\left(\varepsilon_{y 0}\left|\varepsilon_{1}\right|-\frac{\varepsilon_{y 0}^{2}}{2}\right) \quad\left(\right.$ if $\left.\left|\varepsilon_{1}\right| \geq \varepsilon_{y 0}\right)$,

where

$\alpha=\frac{43}{40} \quad 0 \leq a \leq 0.8, \quad \alpha=\frac{7}{10} \quad 0.8<a \leq 1$.

\section{Numerical examples of the optimal design}

To examine the validity of the present algorithm, four numerical examples are shown in this section. The first two examples deal with a simple straight beam and an " $L$ "-shaped beam. The last two deal with rather complicated frame structures and one is subject to the prescribed displacement while the other is subjected to the prescribed load.

\subsection{Example 1}

For the first example, a cantilever with a concentrated load at the free end as shown in Fig. 14 was calculated. The volume constraint was set to $\Omega_{0} / \Omega=0.5$. The beam was equally divided into 10 elements which have 10 layers each and the prescribed displacement $w=1 \mathrm{~mm}$ in the transverse direction was incrementally given at the free end. The material was uniformly distributed first and an iteration for optimization was made after each time $w$ reached $1 \mathrm{~mm}$ until the solution converged. As the criterion of the convergence of optimization, the following condition was employed:

$\frac{U_{\mathrm{ext}}^{(n+1)}}{U_{\mathrm{ext}}^{(n)}}=10^{-4}$,

where $U_{\text {ext }}^{(n)}$ is the external work done by the applied load in the $n$-th iteration. It should be noted that the present algorithm optimizes a design so that the external work may be maximum in this case where a prescribed displacement is given instead of a prescribed load. The history of the loaddisplacement curve is shown in Fig. 15; $P_{y}=\left(t^{2} \sigma_{y}\right) / 4$ is the load which gives the full plastic moment at the fixed end. It can be observed that the optimally designed beam has almost the same strength as the solid beam although it has only half of the material inside. Figure 16 depicts the history of the external work done by the prescribed displacement, where the external work is normalized by the external work of the initial design. For comparison, the result when the material is elastic is also shown. It can be observed that external work increases drastically and almost converges in the first six iterations. Unlike the case of the linear analysis, the history of the external work does not monotonously increase. This is due to the discrepancy of $\partial E / \partial a$ at $a=0.8$ as shown in Fig. 11. It can be corrected by employing the continuous function for $\partial E / \partial a$. Figure 17 shows the history of the material distribution in the beam. In dark layers, the cavity of the microstructure is small, i.e. the material is close to solid. It is observed that the material distribution at the 5-th iteration is very similar to that of the converged solution. In the converged solution, the solid and the hollow regions are clearly separated. For comparison, the optimal configuration in the elastic analysis is shown in Fig. 18. The main difference is that the material is not fully filled near the fixed end since a plastic hinge is not conformed at the fixed end in the elastic analysis.

\subsection{Example 2}

For the second example, the " $L$ "-shaped beam shown in Fig. 19 was calculated. The prescribed displacement $w=1 \mathrm{~mm}$ was also given at the free end. The volume constraint was set to $\Omega_{0} / \Omega=0.7$. Both the vertical and horizontal members are divided into 10 elements, respectively, with 8 layers. As 


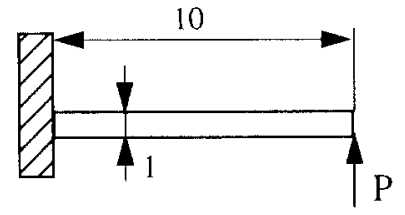

Fig. 14. A cantilever subjected to a concentrated load

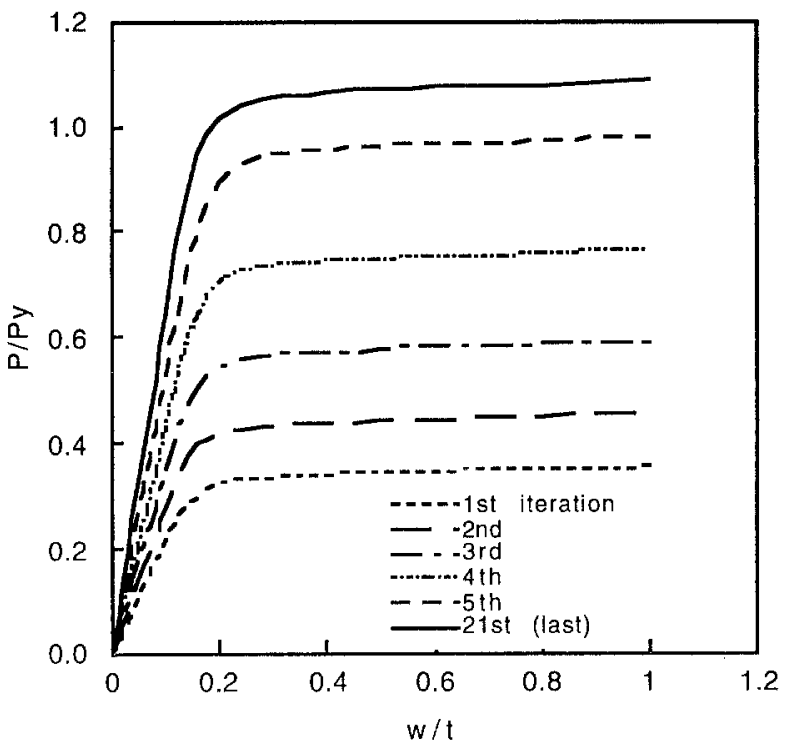

Fig. 15. History of the load-displacement curve of a cantilever with a concentrated load

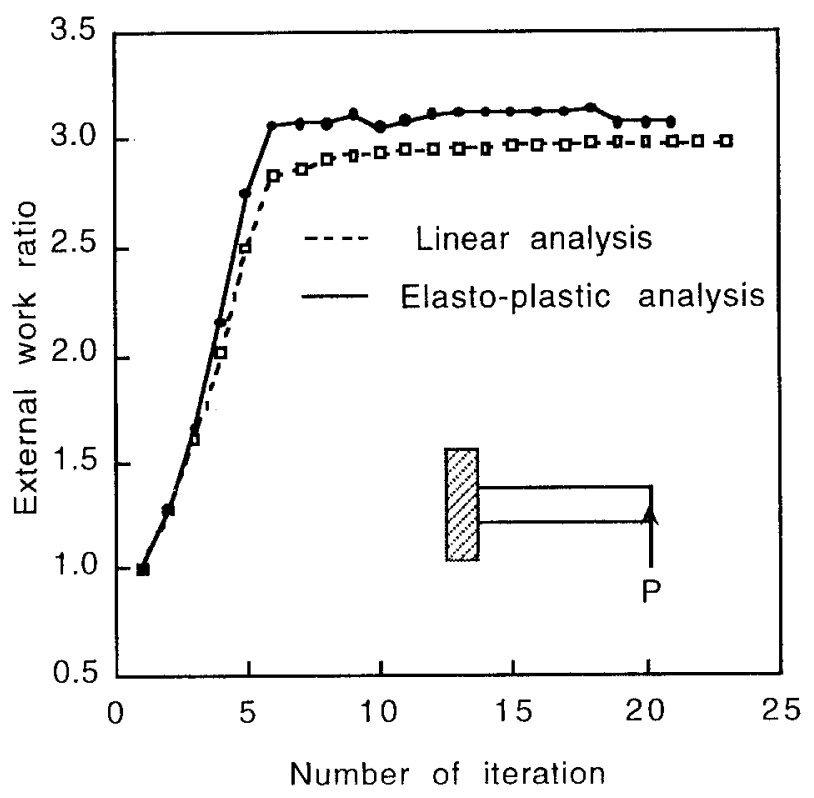

Fig. 16. History of the external work of a cantilever

shown in Example 1, a design improves rapidly in the early iterations and changes little in the latter iterations. Thus the convergence condition $U_{\text {ext }}^{(n+1)} / U_{\text {ext }}^{(n)}$ was changed from $10^{-4}$ in the example to $10^{-3}$. The history of the external work is shown in Fig. 20, where it can be observed that the convergence is very rapid and the number of iterations reduced to 13 . Figure 21 depicts the history of the load-displacement

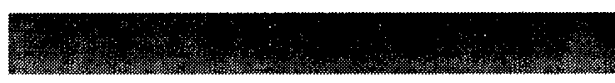

(a) Initial iteration

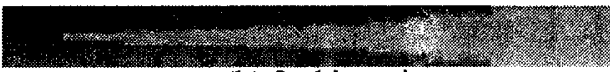

(b) 2nd iteration

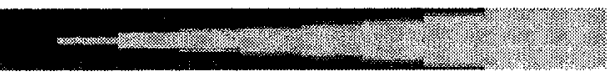

(c) 3rd iteration

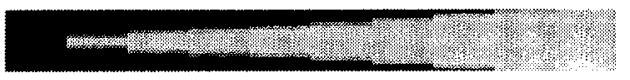

(d) 4th iteration

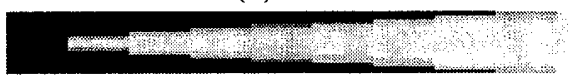

(e) 5 th iteration

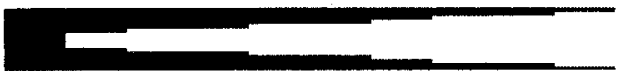

(f) 21 st(last) iteration

Fig. 17. Optimal design of a cantilever subjected to a plastic deformation

Fig. 18. Optimal design of an elastic cantilever

curve. The curve of the 5 th iteration agrees well with that of the 13th iteration. Figure 22a shows the optimal design obtained and the area yielded at $w=1 \mathrm{~mm}$ for the optimal design is shown in Fig. 22b, where a black area expresses plastic deformations occurring while a gray area is elastic. Since a small deformation is assumed in the present formulation for frame structures, the material distribution and the area yielded of a vertical member is uniform in the longitudinal direction. However, the material is not symmetrically distributed to the neutral axis in the vertical member due to the effect of the axial force while it is distributed symmetry in the horizontal member. In rather complex frame structures, the asymmetry to the neutral axis of bending may cause failure to obtain the converged solution. In the following two examples, the material distribution is decided so that the symmetry to the neutral axis may be kept. In these examples, the same cavity size of the microstructure was employed for both layers which are at the same distance from the neutral axis by taking a mean value of the internal work.

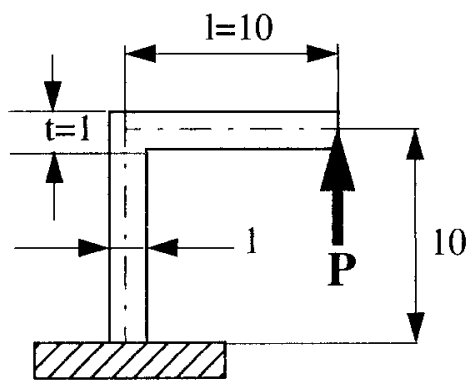

Fig. 19. An $L$-shaped beam with a concentrated load 


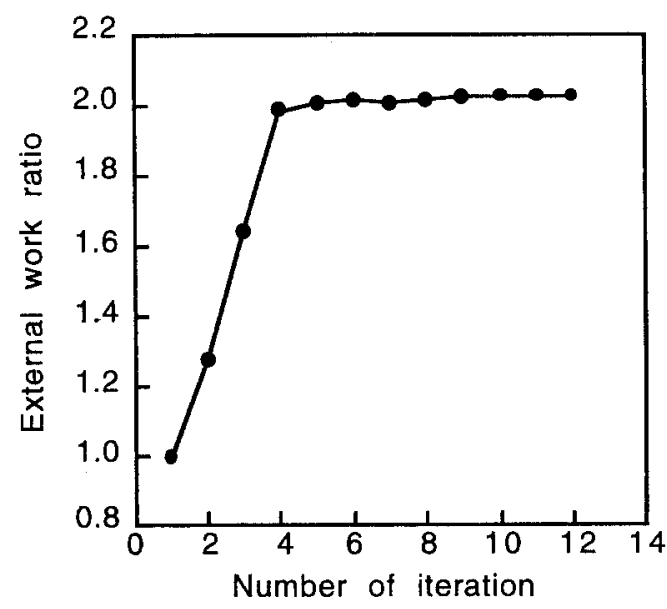

Fig. 20. History of the external work of an $L$-shaped beam

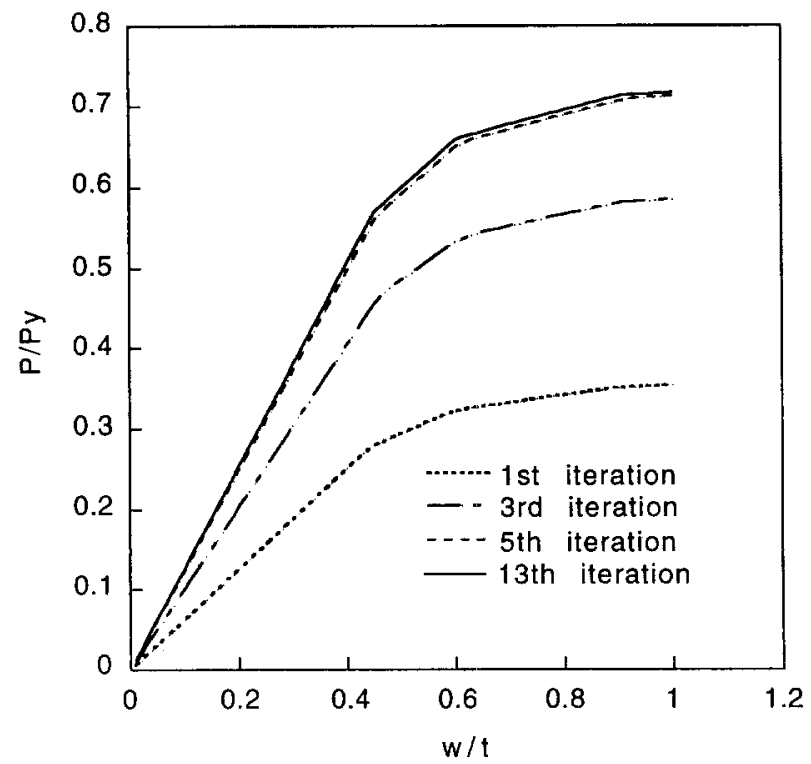

Fig. 21. History of the load-displacement curve of an $L$-shaped beam

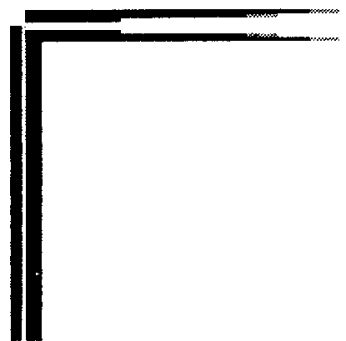

(a) Optimal design

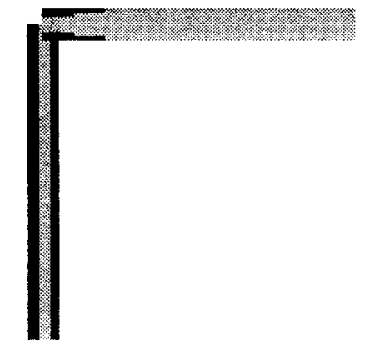

(b) Yielded area
Fig. 22. Optimal design of a frame with a concentrated load

\subsection{Example 9}

For the third example, a problem shown in Fig. 23, where a frame is subjected to a concentrated vertical load, was calculated. As mentioned in the previous example, the material was distributed symmetrically to the neutral axis in this example. The volume constraint is set to $\Omega_{0} / \Omega=0.5$. The frame was divided into 30 equal elements with 8 layers and the prescribed displacement $w=1 \mathrm{~mm}$ was given. The convergence criterion is the same as that of Example 2. The histories of the external work is shown in Fig. 24. It can be observed that the external work done by the prescribed displacement finally becomes nearly three times that of the initial design. The history of the load-displacement curve is shown in Fig. 25, where the collapse load for the case of $a$ $=1$ (solid) by the limit analysis is shown as $P_{\max }$. It can be observed that the optimal design has the same strength as that of the fully solid structure. Due to the shear stiffness, the present optimal design has rather higher strength than the theoretical collapse load. To obtain good agreement with the theoretical collapse load, the simplest way is to reduce the shear stiffness after the normal stress reaches the yield stress. Another method is to employ the higher order element. Figure 26 shows the optimal design obtained. It can be observed that the material is distributed almost uniformly where the bending moment is nearly zero. Figure $27 \mathrm{~b}$ shows the development of the area yielded for both the initial and optimal designs. In the optimal design, the area yielded spreads widely while it is limited and the typical beam collapse mode takes place in the initial design.

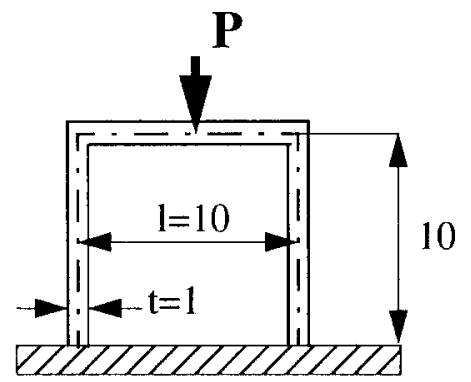

Fig. 23. A frame subject to a vertical load

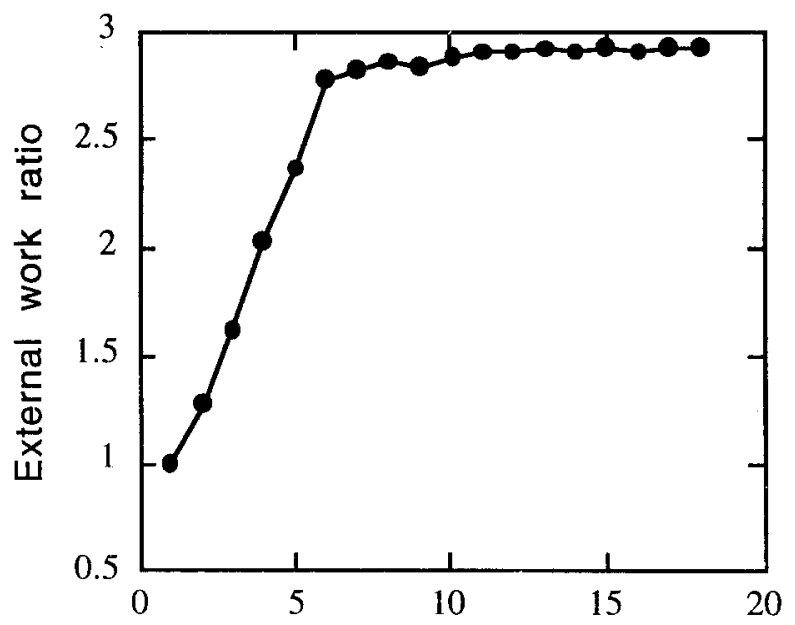

Fig. 24. History of the external work of a frame structure subject to a vertical load

\subsection{Example 4}

As the fourth example, a problem shown in Fig. 28, where a frame is subjected to uniformly distributed loads in the horizontal direction, was calculated. The prescribed loads were given incrementally until the summation of those loads 


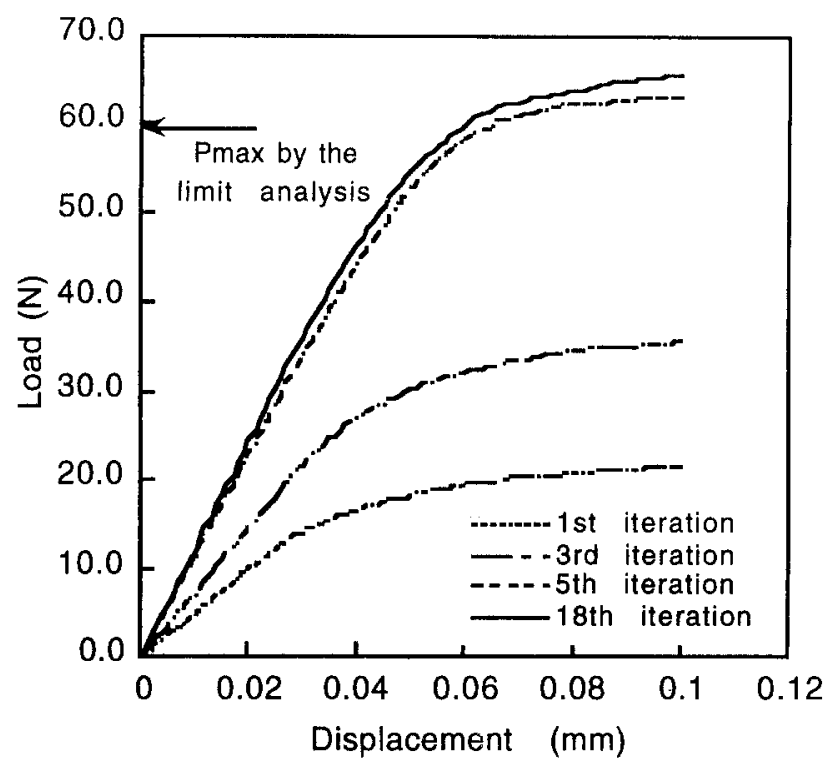

Fig. 25. History of the load-displacement curve of a frame subject to a vertical load

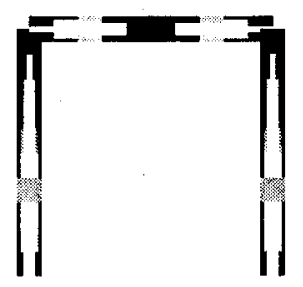

Fig. 26. Optimal design of a frame subject to a vertical load

reached $P=9.8 \mathrm{~N}$. The volume constraint was set to $\Omega_{0} / \Omega$ $=0.5$. It should be noted that the external work should be minimum in this case while it should be maximum in the previous examples where the prescribed displacements were given.

In the initial design where the material was distributed uniformly, the whole area near the fixed end had yielded before the loads reached the prescribed value and the horizontal displacement of the Point $A$ in Fig. 28 became very large since there was little stiffness. As the iteration of optimization went on, the area yielded became smaller and finally plastic deformations took place in a very limited area near the fixed end. The horizontal displacement of $A$ in the optimal design is $0.16 \mathrm{~mm}$ at $P=9.8 \mathrm{~N}$, while it is almost $30 \mathrm{~mm}$ in the initial design. As a result, the external work changes drastically as shown in Fig. 29. Figure 30 shows the relations between the load $P$ and the horizontal displacement until $0.5 \mathrm{~mm}$ are shown. It can be observed that the frame collapsed at a lower load than the prescribed load in the early iterations. The collapsed load at the first iteration (the initial design) is about $2.5 \mathrm{~N}$ while it is over $10 \mathrm{~N}$ in the optimal design. Figure 31 shows the history of the material distribution. Since the structure is optimized according to the normal strain, the element where a bending moment and an axial force are very small but a shear force is large has little material. This may not be a practical design. This would be corrected by setting the least value of the material volume for each layer of an element. It can be also corrected
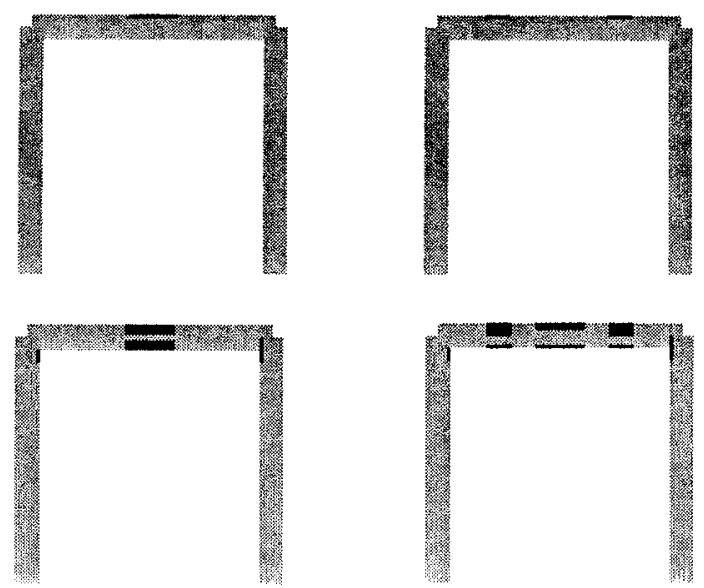

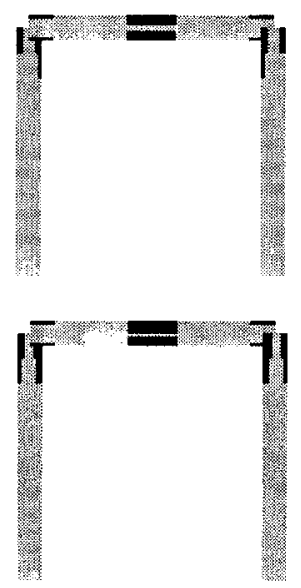

(a) Initial design
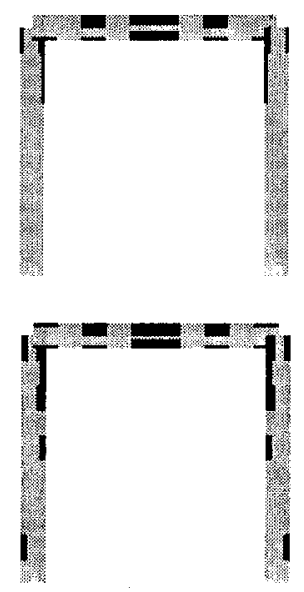

(b) Optimal design
Fig. 27. History of plastification of a frame subject to a vertical load

by taking a shear deformation into the formulation. As far as the shear strain is assumed to be independent of the normal strain, the present algorithm can be extended to include a shear deformation without any difficulty. Figure 32 depicts the area yielded at $P=9.8 \mathrm{~N}$. In the early iterations, plastic deformations occur widely but the area becomes very small in the optimal design while the yielded area spread wider and wider by an iteration in the previous calculations where the prescribed displacements were given.

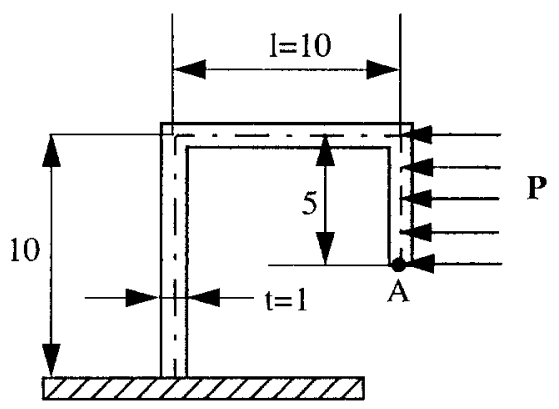

Fig. 28. A frame subjected to horizontal loads 


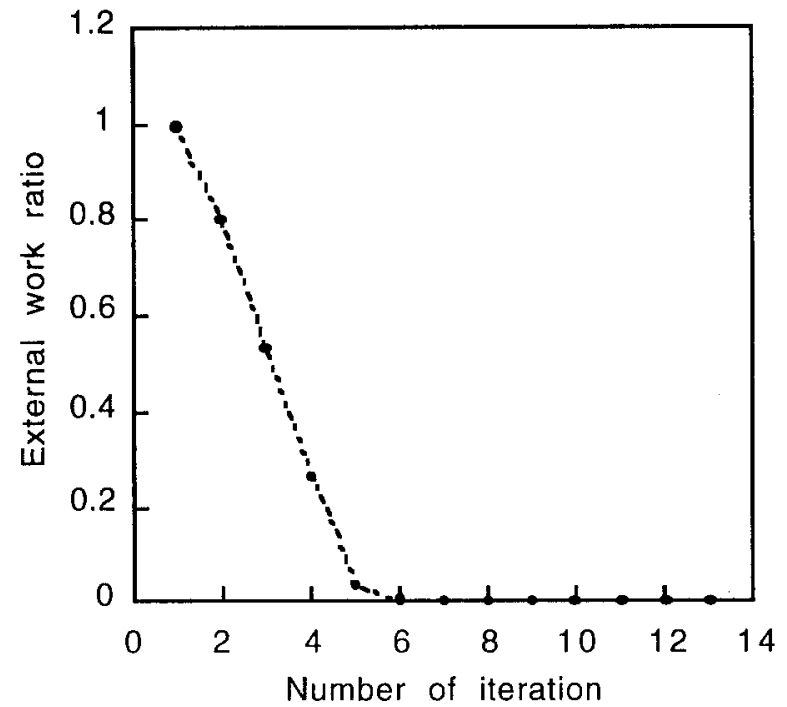

Fig. 29. History of the external work of a frame subjected to horizontal loads

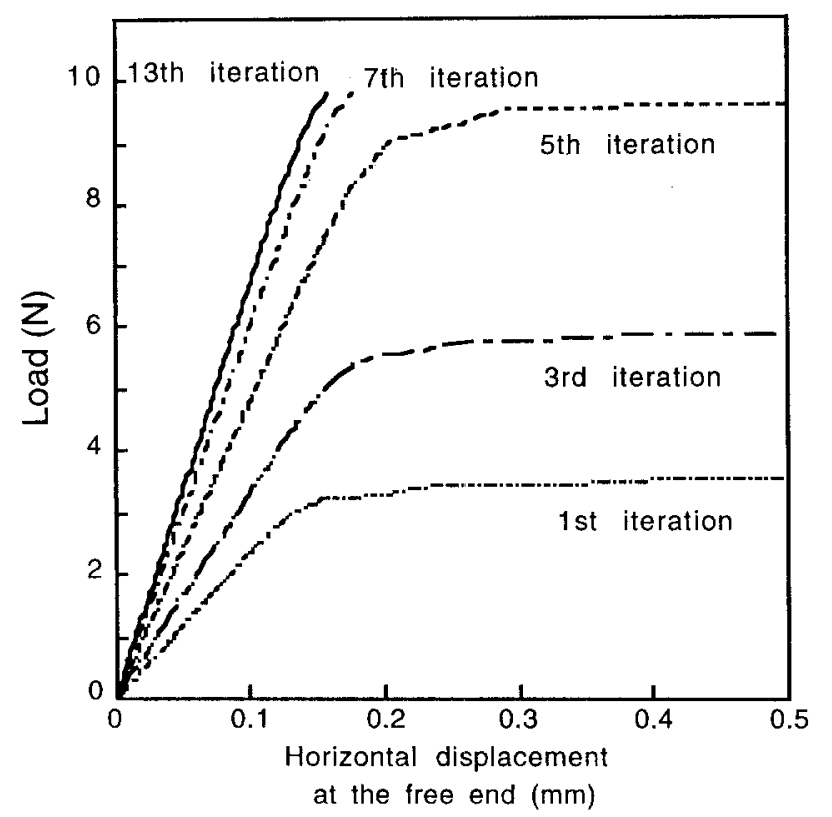

Fig. 30. History of the load-displacement curve of a frame subjected to horizontal loads

\section{Discussion}

The effectiveness of the present algorithm has been demonstrated through several numerical examples in the previous section. Since the effect of shear deformations is not so important for most frame structures, the present algorithm in which the effect is neglected would give a practical optimal design. It may be better, however, to consider shear deformations in the optimization of those structures composed of short members. The simplest method to consider the effect is to assume that the shear modulus in (20) is a function of the cavity size as $G=G^{H}(a)$ and that it is independent of the longitudinal modulus $E_{0}^{H}(a)$. In the following, a formulation with this assumption is discussed.

Since the shear rigidity should become rapidly small as the cavity size becomes large in the present microstructure,

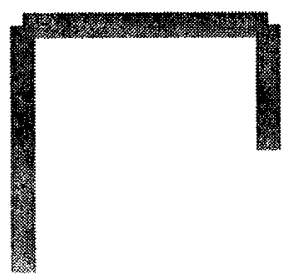

(a) 1st iteration

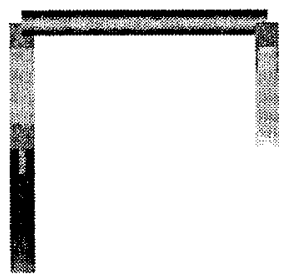

(b) 3rd iteration

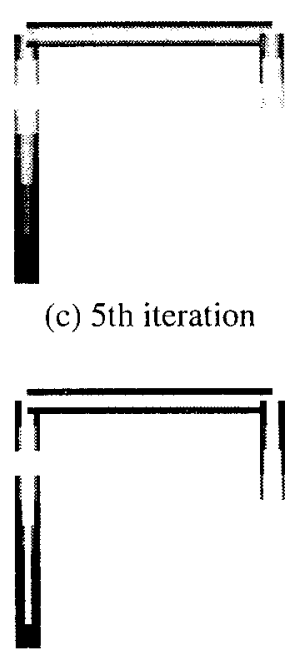

(d) 13th iteration

Fig. 31. Material distribution of a frame subjected to horizontal loads

we assume $G^{H}(a)$ as

$G^{H}(a)=a^{2} G_{0}$.

Where $G_{0}$ is the shear rigidity for $a=1$ (solid). Substituting (44) into (40) and taking differentiation with respect to $a_{\ell}$, we obtain the following sensitivity instead of (41):

$$
\begin{aligned}
& \frac{\partial U_{\text {int }}}{\partial a_{\ell}}=\alpha E_{0} \frac{\varepsilon_{1}^{2}}{2}+a_{\ell} G_{0} \gamma_{1}^{2} \quad\left(\text { if }\left|\varepsilon_{1}\right|<\varepsilon_{y 0}\right), \\
& \frac{\partial U_{\text {int }}}{\partial a_{\ell}}=\alpha E_{0}\left(\varepsilon_{y 0}\left|\varepsilon_{1}\right|-\frac{\varepsilon_{y 0}^{2}}{2}\right)+a_{\ell} G_{0} \gamma_{1}^{2} \quad\left(\text { if }\left|\varepsilon_{1}\right| \geq \varepsilon_{y 0}\right),
\end{aligned}
$$

Example 4 in the previous section was calculated with the modified algorithm. As shown in Fig. 33 the optimal design obtained is similar to the one calculated with the original algorithm shown in Fig. 31d except area $B$, where there exists little bending moment and therefore little material is distributed by the original algorithm. Figure 34 shows the comparison of the load-displacement curves of the optimal 


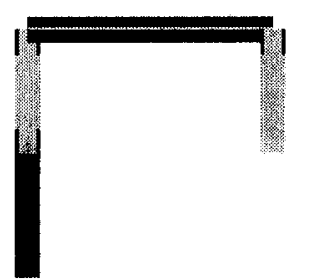

(a) 1st iteration

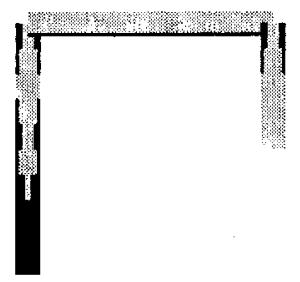

(b) 3rd iteration

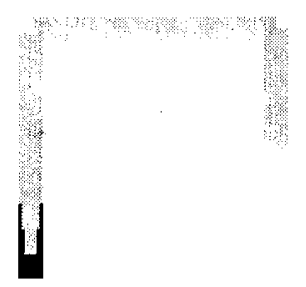

(c) 5th iteration

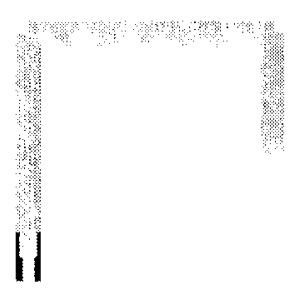

(d) 13th iteration

Fig. 32. Yielded area of a frame subjected to horizontal loads

designs obtained with the original and modified algorithms. There is no significant difference between them.

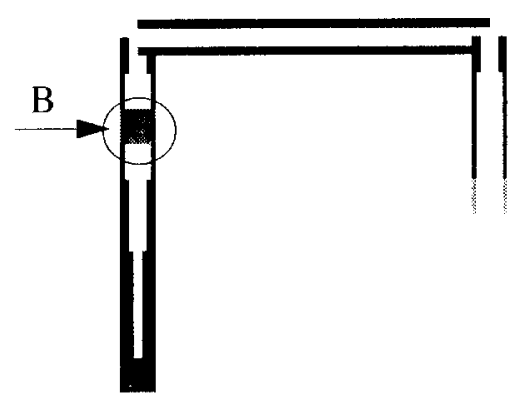

Fig. 33. The optimal design obtained with the modified algorithm

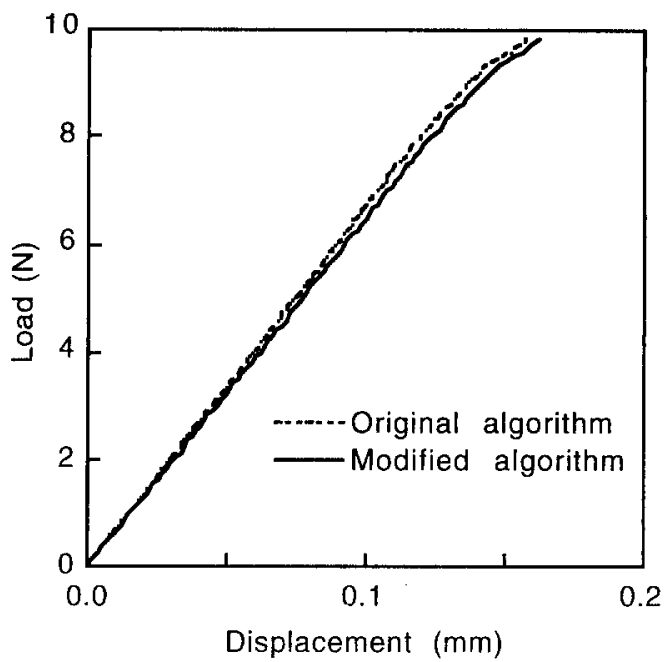

Fig. 34. Load-displacement curves obtained with the modified algorithm

\section{Conclusion}

The generalized layout optimization method proposed by Bendsøe and Kikuchi (1988) was extended to the optimization of a frame structure subjected to a plastic deformation. In the present formulation, the domain in which a material can be distributed is limited inside frame members and optimization is conducted so that the external work done by the prescribed load may be minimum. Through the numerical examples, it was proven that the present algorithm gives a much improved design compared to the initial design and it converges well.

\section{References}

Bendsøe, M.P.; Kikuchi, N. 1988: Generating optimal topologies in structural design using a homogenization method. Comp. Meth. Appl. Mech. \& Engrg. 71, 197-224

Diaz, A.R.; Kikuchi, N. 1992: Solutions to shape and topology eigenvalue optimization method. Int. J. Num. Meth. Engrg. 35, 1487-1502

Guedes, J.M.; Kikuchi, N. 1990: Preprocessing and postprocessing for materials based on the homogenization method with adaptive finite element methods. Comp. Meth. Appl. Mech. \& Engrg. 83, $143-198$

Haftka, R.T.; Grandhi, R.V. 1986: Structural shape optimizationA survey. Comp. Meth. Appl. Mech. EEngrg. 57, 91-106

Hughes, T.J.R. 1977: A simple and efficient finite element for plate bending. Int. J. Num. Meth. Engrg. 11, 1529-1543

Ma, Z.D.; Kikuchi, N.; Hagiwara, I. 1993: Structural topology and shape optimization for a frequency response problem. Comp. Mech. 13, 157-174

Owen, D.J.R.; Hinton, E. 1980: Finite elements in plasticity. Swansea: Pineridge Press

Suzuki, K.; Kikuchi, N. 1991: A homogenization method for shape and topology optimization. Comp. Meth. Appl. Mech. GS Engrg. 92, 291-318 